\title{
PATTERN CLASSIFICATION BY SPIKING NEURAL NETWORKS COMBINING SELF-ORGANIZED AND REWARD-RELATED SPIKE-TIMING-DEPENDENT PLASTICITY
}

\author{
Sou Nobukawa ${ }^{1, *}$, Haruhiko Nishimura $^{2}$, and Teruya Yamanishi ${ }^{3}$ \\ ${ }^{1}$ Department of Computer Science, Chiba Institute of Technology \\ 2-17-1 Tsudanuma, Narashino, Chiba, 275-0016 Japan \\ ${ }^{2}$ Graduate School of Applied Informatics, University of Hyogo \\ 7-1-28 Chuo-ku, Kobe, Hyogo, 650-8588 Japan \\ ${ }^{3}$ Department of Management and Information Sciences, Fukui University of Technology \\ 3-6-1 Gakuen, Fukui, Fukui, 910-8505 Japan \\ *E-mail: nobukawa@cs.it-chiba.ac.jp
}

Submitted: 16th March 2019; Accepted: 12th May 2019

\begin{abstract}
Many recent studies have applied to spike neural networks with spike-timing-dependent plasticity (STDP) to machine learning problems. The learning abilities of dopaminemodulated STDP (DA-STDP) for reward-related synaptic plasticity have also been gathering attention. Following these studies, we hypothesize that a network structure combining self-organized STDP and reward-related DA-STDP can solve the machine learning problem of pattern classification. Therefore, we studied the ability of a network in which recurrent spiking neural networks are combined with STDP for non-supervised learning, with an output layer joined by DA-STDP for supervised learning, to perform pattern classification. We confirmed that this network could perform pattern classification using the STDP effect for emphasizing features of the input spike pattern and DA-STDP supervised learning. Therefore, our proposed spiking neural network may prove to be a useful approach for machine learning problems.
\end{abstract}

Keywords: spiking neural network, spike timing-dependent plasticity, dopamine-modulated spike timing-dependent plasticity, pattern classification

\section{Introduction}

Different types of neural coding (including rate, temporal, and population coding) support adaptive brain information processes $[1,2,3]$. Spiking neural networks can describe these types of neural codes based on the spiking activity generated from membrane potential dynamics and have therefore been widely utilized to reveal the mechanisms for information processes such as memory and learning $[4,5,6,7,8]$. Recent studies have reported the application of these spiking neural networks to machine learning $[9,10,11,12,13,14,15,16,17,18]$.

Previously, spiking neural networks had difficulty in learning by conventional backpropagation, which is used in the rate coding model. This difficulty was attributed to the fact that the spikes, in- 
cluding the discontinuous trajectory of the ystem state, are not differentiable [19]; however, differentiable activation functions are vital for conventional backpropagation. To overcome this difficulty, Lee et al. proposed a method to adopt backpropagation into the spiking neural network, focusing on the continuous membrane potential behaviors [10]. Subsequently, they trained deep spiking neural networks using this backpropagation [10]. However, their backpropagation only focused on the temporal dominant spike behaviors and ignored the spatial dominant spike behaviors. Moreover, to enhance the accuracy and efficiency of learning, Wu et al. expanded the backpropagation for spiking neural networks, considering both spatiotemporal and temporal behaviors of spikes [18].

As another approach for learning for spiking neural networks, a learning approach using physiological spike-timing-dependent plasticity (STDP) has been used. For example, Kasabov et al. proposed a spiking neural network for classification and time-series prediction, called NeuCube $[9,20$, $21,22]$. NeuCube is composed of an encoding module as the input layer, spiking neural clusters located in three-dimensional space as the inter-layer and a function module with supervised learning as the output layer. Another example of spiking neural networks for classification is the convolutional spiking neural network, composed of convolutional and pooling layers with STDP, proposed by Kheradpisheh et al. [13]. However, in their network, classification is implemented using a support vector machine located at the output layer. In addition, Tavanaei et al. proposed new STDP rules based on backpropagation in the fully connected network as output layers in a convolutional neural network [17]. In their study, a convolutional neural network could be implemented using a spiking neural network alone, rather than using the conventional rate coding model. A liquid state machine, which is a spiking network consisting of an input layer, a recurrent intermediate layer called a reservoir, and a readout layer is also widely utilized for dynamical pattern classification [23, 24, 25, 26]. Recently, Jin $\& \mathrm{Li}$ adapted a supervised type of STDP to the liquid state machine and showed that their proposed network exhibited high accuracy for speech recognition [27].
Based on these studies, dopamine (DA) synapses (the neurotransmitter involved in rewardrelated synaptic plasticity), can be adopted to the learning method for the spiking neural networks $[28,29,30,31,32,33,16]$. In STDP rules, the synaptic weights are constructed by inter-network spikes. However, in dopamine-modulated STDP (DA-STDP) rules, they are constructed based on inter-network spikes and reward signals that correspond to teaching signals. In brief, DA-STDP and STDP correspond to supervised and unsupervised types of synaptic plasticity, respectively. As an example of an application of DA-STDP, Mazafari et al. showed that DA-STDP exhibits a greater ability to learn objective recognition (supervised learning), compared to STDP for unsupervised learning [16]. Moreover, as an example of a spiking neural network combining STDP and DA-STDP, Kawai et al. and Warlaumont et al. demonstrated that their proposed networks acquire a motor control function, and STDP applying a feedback signal from the objective system enhances the learning efficiency of DA-STDP $[32,33]$. Their proposed spiking neural networks added the synaptic plasticity in the reservoir layer to the conventional structure of the liquid state machines [23, 24, 25, 26]. However, they did not consider learning for many motor patterns. Therefore, the learning ability of the spiking neural networks combined with STDP and DA-STDP against several tasks has not been studied. The applicability of this network to other machine learning problems has also not been clarified. Hence, in this study, we examine the classification for numerous patterns as a machine learning problem, using a spiking neural network that combines STDP and DA-STDP.

\section{Materials and Methods}

\subsection{Spiking neural network}

Figure 1 shows the structure of the spiking neural network used in this study. This spiking neural network comprises two types of sub-networks. The first is a recurrent neural network that consists of excitatory neurons, with synapses self-organized by STDP and inhibitory neurons. As the input neural population, a part of the excitatory neural population is joined by $N_{\text {ex }}$ input spike generators with the Poisson process. The joined probability to excita- 
tory neurons was set to 0.1 . The combination of activated (spike rate $\left.\Lambda_{i}>0[\mathrm{~Hz}]\left(i=1,2, \cdots, N_{\mathrm{ex}}\right)\right)$ and deactivated states $\left(\Lambda_{i}=0[\mathrm{~Hz}]\right)$ in this generator represents the input pattern. The second subnetwork is the neural population that consists of excitatory neurons as an output layer. These excitatory neurons are joined to excitatory neurons in the previous layer by DA-STDP synapses. Here, the jointed probability of excitatory neurons between layers is set to 0.01 . With this network structure, we expect that the spikes induced by the input spike generators propagate through the recurrent neural network and reach the output layer. This propagation is emphasized by STDP and DA-STDP learning.

In this spiking neural network, the membrane potential $v(t)$ of each neuron is described by the conductance based on a leaky-integrate-and-fire neuron model

$$
\begin{gathered}
\frac{d v}{d t}=-\frac{1}{\tau_{m}}\left(v-V_{L}\right)-g_{E}\left(v-V_{E}\right)-g_{I}\left(v-V_{I}\right), \\
\text { if } v \geq V_{\mathrm{thr}}[\mathrm{mV}], \text { then } v(t) \rightarrow V_{r},
\end{gathered}
$$

here $\tau_{m}, V_{E}, V_{I}$, and $V_{L}$ are the membrane decay constant, reversal potentials of the AMPAreceptor-mediated excitatory synaptic current, inhibitory synaptic current, and leak current, respectively. The excitatory synaptic conductance $g_{E}(t)$ $\left[\mathrm{ms}^{-1}\right]$ and inhibitory synaptic conductance $g_{I}(t)$ $\left[\mathrm{ms}^{-1}\right]$ are given by

$\frac{d g_{X}}{d t}=-\frac{g_{X}}{\tau_{s}}+\sum_{j} G_{X, j} \sum_{s_{j}} \delta\left(t-s_{j}-d_{j}\right), \quad X=E, I$.

here, $\tau_{s}$ is the decay constant of the excitatory and inhibitory synaptic conductance. $s_{j}, d_{j}, G_{E, j}$, and $G_{I, j}$ are spike times of synaptic input from the $j$-th neuron, synaptic delays, synaptic weights of excitatory, and inhibitory synapses, respectively. In this study, we used parameter sets for $V_{I}=-80[\mathrm{mV}]$, $V_{L}=-70[\mathrm{mV}], V_{r}=-60[\mathrm{mV}], V_{\mathrm{thr}}=-50[\mathrm{mV}]$, $V_{E}=0[\mathrm{mV}], \tau_{m}=20[\mathrm{~ms}]$ (excitatory neuron), $\tau_{m}=10$ [ms] (inhibitory neuron), and $\tau_{s}=2$ [ms]. We solve Eqs. (1), (2), and (3) by the Euler method with the size of time step $\Delta t=0.1[\mathrm{~ms}]$. The refractory period is set to 1 [ms]. In excitatory-toexcitatory connections and other connections, the synaptic delays are set to uniform random values between 1 to $3[\mathrm{~ms}]$ and ones between 0 to 2 [ms]. The size of spiking neural networks with STDP is
$N_{E}=10000$ and $N_{I}=2000$ for excitatory neurons and inhibitory neurons, respectively. The size of the neural population at the output layer is set to $N_{o}=10$.

The synaptic weights $G_{E, j}$ in the spiking neural network are self-organized by the STDP rule as follows

$$
\begin{aligned}
& \Delta G= \begin{cases}A_{+} e^{-t_{\text {stdp }} / \tau_{+}} & \left(\text {if } t_{\text {stdp }}>0\right), \\
A_{-} e^{-t_{\text {stdp }}} / \tau_{-} & \left(\text {if } t_{\text {stdp }}<0\right),\end{cases} \\
& t_{\text {stdp }}=t_{\text {post }}-t_{\text {pre }} \text {, }
\end{aligned}
$$

here, $t_{\text {post }}$ and $t_{\text {pre }}$ are the spike times of the post- and pre-neuron, respectively. The parameters $\left(A_{ \pm}, \tau_{ \pm}\right)$ are set to $A_{+}=0.1, A_{-}=0.12$, and $\tau_{ \pm}=20$ [ms][34].

The output layer is jointed to the recurrent spiking neural network by the synaptic connections with DA-STDP [35]. DA-STDP rule is given by

$$
\begin{aligned}
\dot{c}_{\mathrm{da}} & =-c_{\mathrm{da}} / \tau_{c}+\operatorname{STDP}\left(t_{\mathrm{stdp}}\right) \delta\left(t-t_{\mathrm{pre} / \mathrm{post}}\right)(6) \\
\dot{d} & =-d / \tau_{d}+\operatorname{DA}(t), \\
\dot{w}_{\text {out }} & =d P_{\mathrm{da}} \dot{c}_{\mathrm{da}},
\end{aligned}
$$

here, $c_{\mathrm{da}}, d, \tau_{c, d}$, and $P_{\mathrm{da}}$ represent the degree of activation for an enzyme for plasticity, DA concentration $[\mu M]$, time constants, and learning rate, respectively. $\operatorname{STDP}\left(t_{\text {stdp }}\right)$ is the STDP function given by Eq.(4). $\mathrm{DA}(t)$ is the reward signal, i.e., $\mathrm{DA}(t)=1,0$ in the case for applying the reward or not. In this study, we used $P_{\mathrm{da}}=0.01, \tau_{c}=200[\mathrm{~ms}]$ and $\tau_{d}=2$ [ms] [33].

\subsection{Evaluation indexes}

To observe neural activity, we used spiking rates in the excitatory neural population $r_{E} \mathrm{~Hz}$ and the inhibitory neural population $r_{I} \mathrm{~Hz}$ as follows

$$
r_{X}(t)=1000 \frac{S_{X}(t)}{\Delta t N_{X}} \quad X=E, I,
$$

here, $S_{E}$ and $S_{I}$ indicate the counts of spikes in the bin where the width is 0.1 [ms] for excitatory and inhibitory neural populations, respectively. The $r_{E}(t)$ and $r_{I}(t)$ are smoothed by a Gaussian-shaped window with a width of $10[\mathrm{~ms}]$.

\subsection{Learning patterns and timeline for learning and testing}

Figure 2 shows the input spiking patterns for learning and the timeline for learning and testing. 


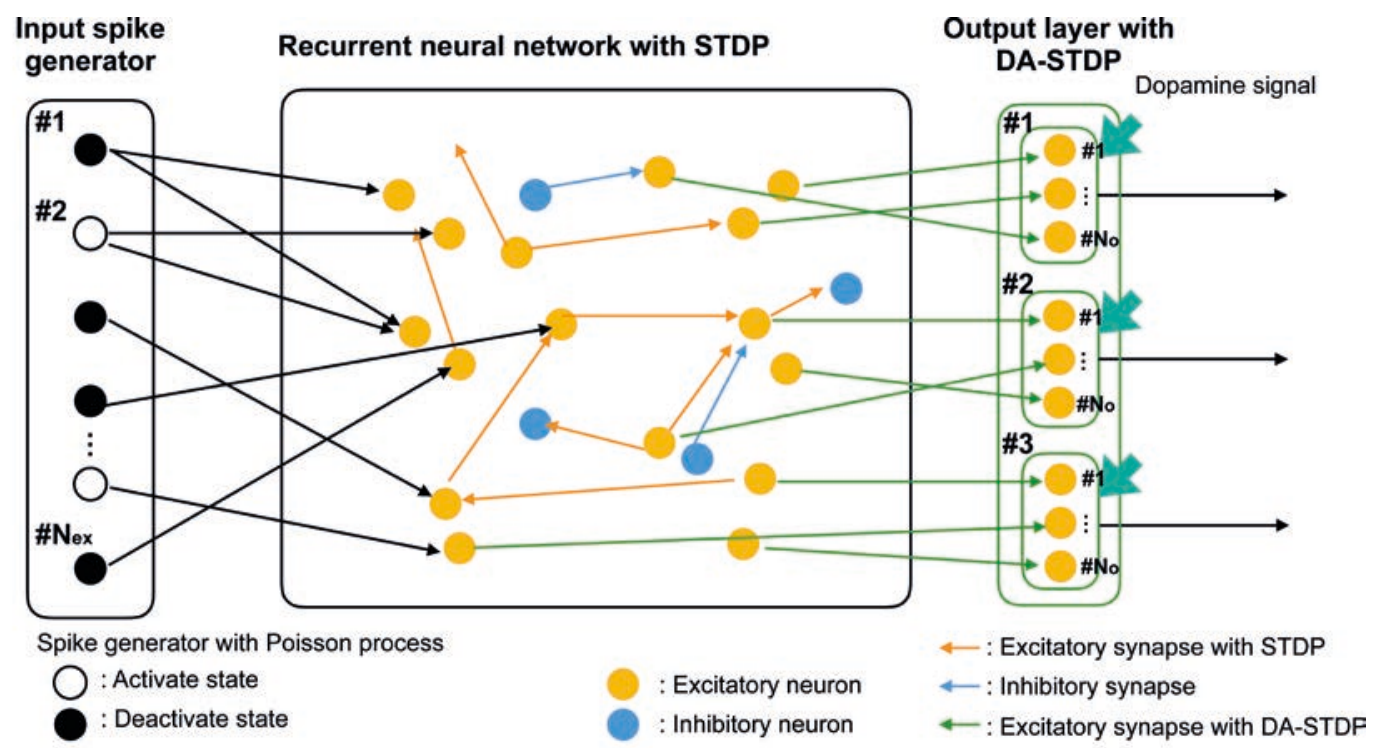

Figure 1. Spiking neural network comprising mutually connected recurrent network layer with spike-timing-dependent plasticity (STDP) and output neural layer with dopamine-modulated STDP (DA-STDP). The recurrent neural network comprises excitatory neurons, with synapses self-organized by STDP and inhibitory neurons. As the input neural population, a part of the excitatory neural population is joined by input spike generators with the Poisson process. The output layer is the neural population that comprises the excitatory neurons as an output layer that is joined to excitatory neurons in the previous layer by the DA-STDP synapses.

The learning period is set to $0 \leq t \leq 30$ [sec]. Three input patterns are applied separately. The periods for the input patterns are divided such that one second allows for the transition not updating synaptic weights and the following nine seconds allow for learning by updating synaptic weights following STDP and DA-STDP. Here, when input pattern \# $j$ $(j=1,2,3)$ is applied, the dopamine signal $\mathrm{DA}_{j}(t)$ is set to 1 and the other signals are set to 0 . The test period is set to $30 \leq t \leq 60$ [sec]. The spiking rates of the output neural population for each of the three input patterns is then observed. The \# $j$ input pattern is set to $\Lambda_{i}=\Lambda\left(1+(j-1)\left[\frac{N_{\mathrm{ex}}}{3}\right] \leq i \leq j\left[\frac{N_{\mathrm{ex}}}{3}\right]\right)$, where $[\cdot]$ is a Gaussian symbol, and $i$ indicates a number for input spike generation $\left(i=1,2, \cdots, N_{\mathrm{ex}}\right)$. In the test period, the pattern with the longest time that exhibits the highest spiking rate of output neural population corresponding with the pattern is selected as the recalling pattern.

\section{Results}

\subsection{Classification of input spiking patterns}

Figure 3 shows the time series of the spiking rate in the output neural population $r_{o}^{i}(i=1,2,3)$. In the test period $30 \leq t \leq 60[\mathrm{~s}]$, the high spiking rate of the output neural module is induced by the corresponding input spiking pattern. From 10 trials of learning and recalling patterns, the success rate of recalling exhibits 1.0 against all patterns. Figure 4 represents the distribution of synaptic weights between the recurrent neural network and the output layers after learning. This result confirms that specific synaptic weights are enhanced by DA-STDP.

\subsection{Comparison with a case not adopting STDP in a recurrent neural network}

To evaluate the function of STDP in the recurrent neural network, Figure 5 shows the time series of spiking rates in the output neural population $r_{o}^{i}$ $(i=1,2,3)$ when STDP is not adopted in the recurrent neural network and for $\Lambda=1.0[\mathrm{~Hz}]$. During the test $30 \leq t \leq 60[\mathrm{~s}]$, the high spiking rate of the output neural module was not always induced by a 


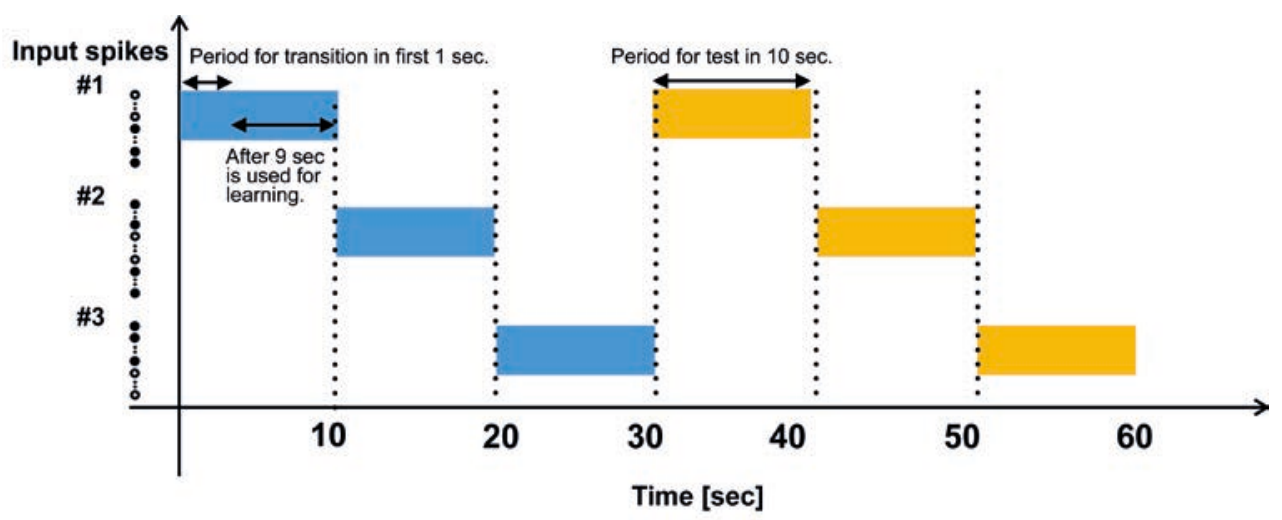

Figure 2. Timeline for learning and evaluation. Blue and orange bars indicate learning and evaluation, respectively. In the learning period, three input patterns are applied separately. The periods for the input patterns are divided such that the first second allows for the transition not updating synaptic weights and

the following nine seconds allow for learning by updating synaptic weights following STDP and DA-STDP. Here, when the input pattern $\# j(j=1,2,3)$ is applied, the dopamine signal $\mathrm{DA}_{j}(t)$ is set to 1 , and the other signals are set to 0 . In the test period, the spiking rates of the output neural population for each of the three input patterns are subsequently observed.
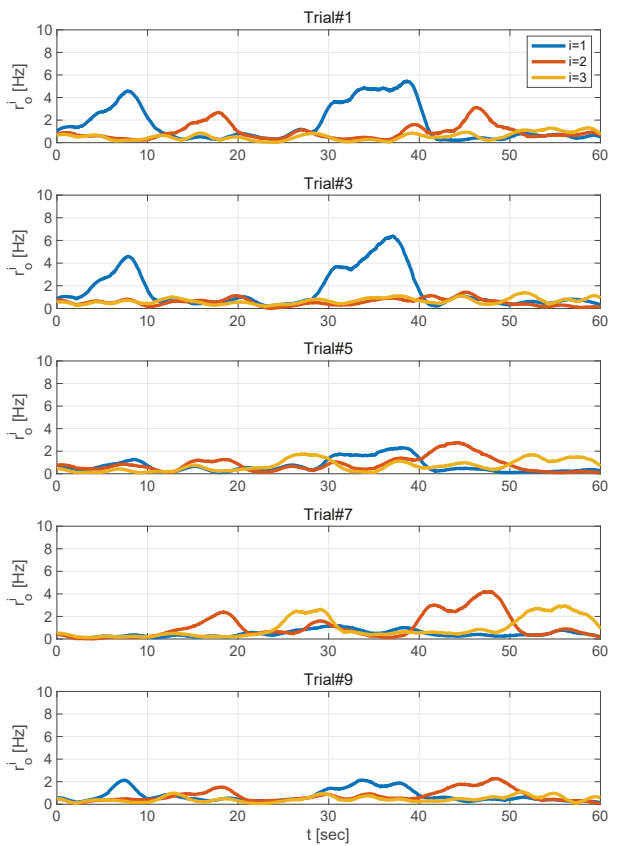
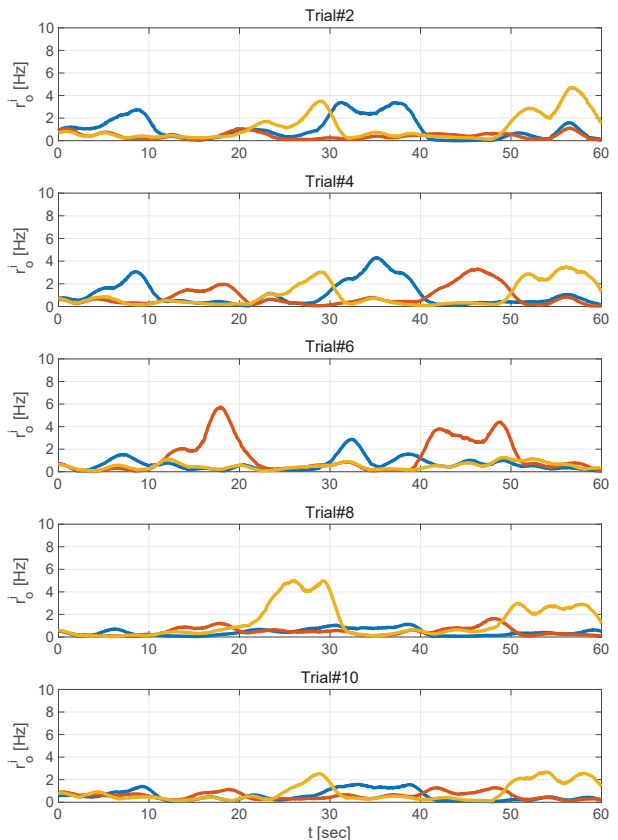

Figure 3. Time series of spiking rates in output neural population $r_{o}^{i}(i=1,2,3)$. In the test period $30 \leq t \leq 60$ [s], the high spiking rate of the output neural module is induced by the corresponding input spiking pattern. From 10 trials of learning and recalling patterns, the success rate of recalling exhibits 1.0 against all patterns. 

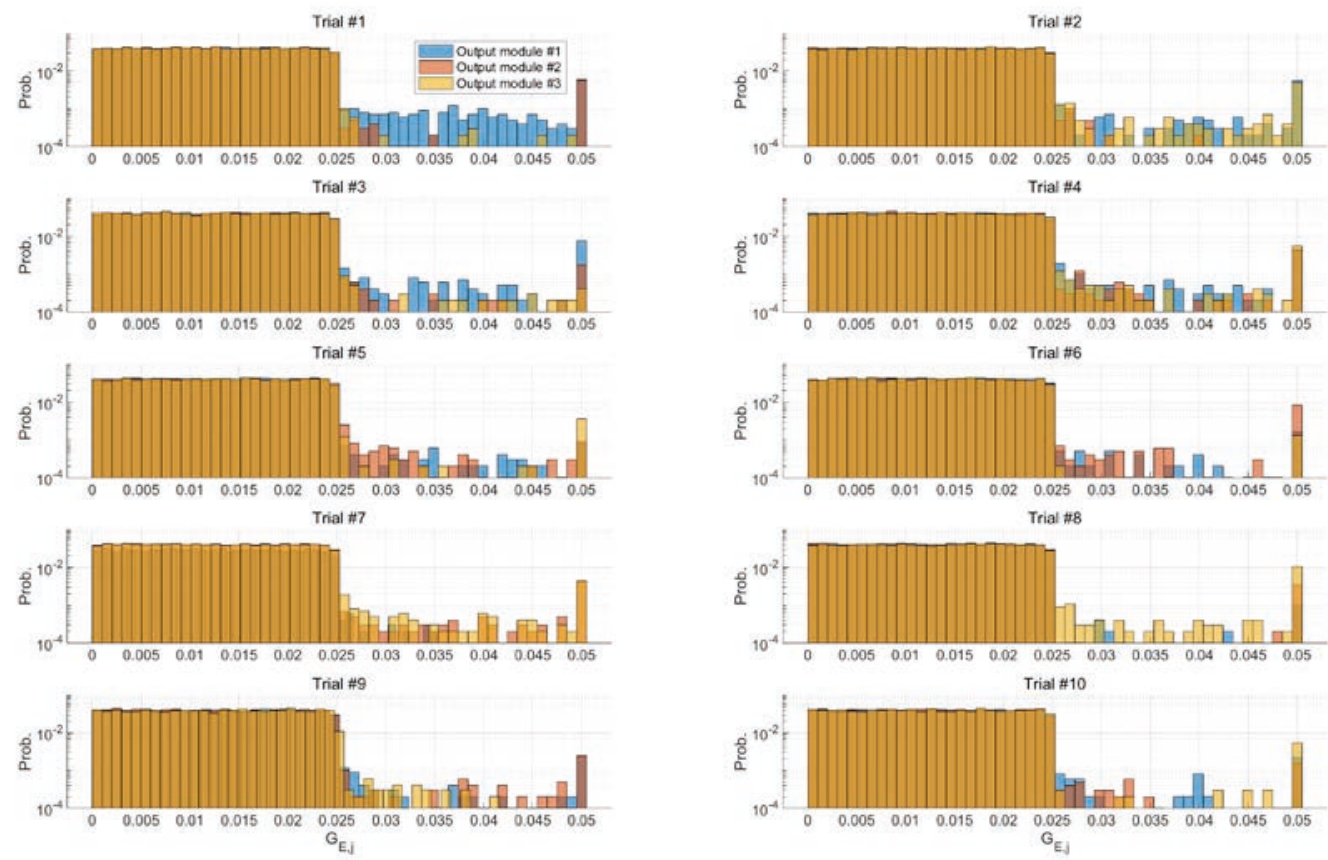

Figure 4. Distribution of synaptic weights between the recurrent neural network and output layers after learning. The synaptic weights specifically enhanced by DA-STDP are confirmed.

corresponding input spiking pattern. From 10 trials of learning and recalling patterns, the success rate of recalling exhibits $0.8,0.9$, and 0.7 against patterns \#1, \#2, and \#3, respectively. In the input spike rate $\Lambda=3.0[\mathrm{~Hz}]$, which corresponds to the input spiking rate of Figures. 3 and 4, the output modules exhibit excessive spiking rates against all input spike patterns, thus showing that pattern classification has not been successful.

\section{Discussion and Conclusion}

In this study we examined the pattern classification abilities of the spiking neural network by combining a recurrent neural network with STDP as self-organized synaptic plasticity, with output modules jointed by DA-STDP as reward-related synaptic plasticity for supervised learning. This network was able to perform pattern classification by learning three input patters. Moreover, when comparing the success rates for recalling patterns between cases applying STDP and not applying STDP, it was found that STDP in recurrent neural networks can enhance the supervised learning by DA-STDP.
The mechanism to obtain the classification function for input spike patterns is as follows. The spikes, which are induced by the input spike generators, propagate through the recurrent neural network. The synaptic weights in this pathway are enhanced by STDP learning. We assume that these pathways for several input spike patterns are different from one another. Further, these enhanced spikes are applied to the output layer through synaptic weights with DA-STDP. By applying the dopamine signal to the output neural population corresponding to the input spike pattern, the synaptic weights from specific pathways are enhanced selectively. Finally, the output neural population produces spikes corresponding to the input spike pattern. Our results show that STDP learning in the recurrent neural network improves success rates for recalling patterns. Moreover, the selectively enhanced synaptic weights between the recurrent network and output layers were observed. Within addition to the learning process, redundant spikes, i.e., the excessive spiking observed in the case of not adopting STDP, are prevented by reducing the unnecessary synaptic weights. It can thus be interpreted that the features of an input spike pattern are emphasized by the self-organized STDP, 

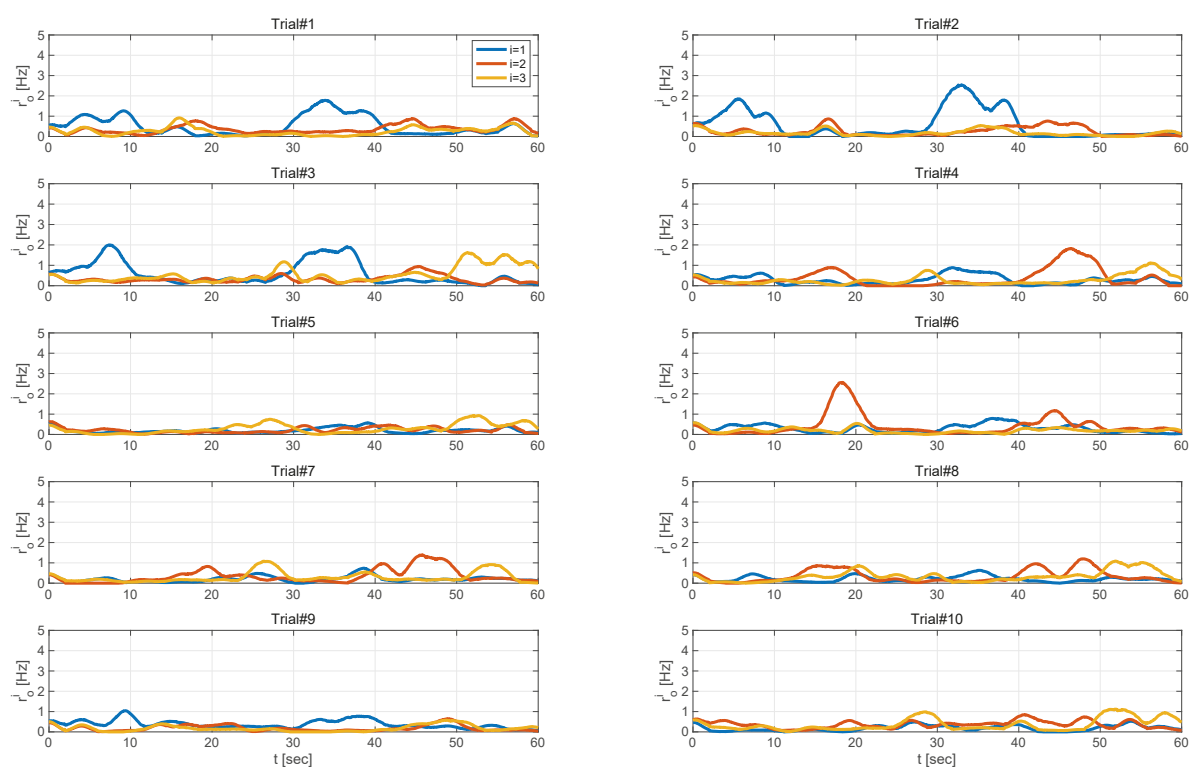

Figure 5. Time series of spiking rate in output neural population $r_{o}^{i}(i=1,2,3)$ not adopting STDP in recurrent neural network and for $\Lambda=1.0[\mathrm{~Hz}]$. Among 10 trials of learning and recalling patterns, the success rate of recalling exhibits $0.8,0.9$, and 0.7 against patterns \#1, \#2, and \#3, respectively.

and consequently, the output layers efficiently learn with DA-STDP based on these features.

Many spiking neural networks adopt a structure in which the spiking neural network with unsupervised learning is located before the output layer with supervised learning $[9,20,21,22,33,13]$. It has been previously shown that convolutional layers with unsupervised STDP synapses can detect the edges of applied images [13] and that selforganized STDP in the recurrent neural network, before output layers, enhance the learning efficiency for motor controls [33]. These findings are congruent with our results.

The main limitation of this study is that the sizes of the input patterns are small (10 spike generators); therefore, the ability to learn and recalling for more expanded sizes should be evaluated in the future. However, in cases with expanding input size, it is assumed that the spiking rates become too high to facilitate proper learning and recall. To prevent the excessive spiking rate, adopting other types of synaptic plasticity, such as intrinsic and short-term plasticity is needed.

In conclusion, the findings presented herein reveal that spiking neural networks combining selforganized and reward-related STDPs have the abil- ity to learn and recall input spike patterns. Although several limitations must be considered, our proposed spiking neural network could be a useful approach for pattern classification in the future.

\section{Acknowledgments}

This work was supported by JSPS KAKENHI for Early-Career Scientists Grant Number 18K18124 (SN) and Grant-in-Aid for Scientific Research (C) Grant Number 18K11450 (TY).

\section{References}

[1] M. I. Rabinovich, P. Varona, A. I. Selverston, and H. D. Abarbanel, Dynamical principles in neuroscience, Reviews of modern physics, vol. 78, no. 4, pp. 1213-1265, 2006.

[2] R. Q. Quiroga and S. Panzeri, Principles of neural coding. CRC Press, 2013.

[3] S. Panzeri, J. H. Macke, J. Gross, and C. Kayser, Neural population coding: combining insights from microscopic and mass signals, Trends in cognitive sciences, vol. 19, no. 3, pp. 162-172, 2015.

[4] N. Schweighofer, K. Doya, H. Fukai, J. V. Chiron, T. Furukawa, and M. Kawato, Chaos may enhance 
information transmission in the inferior olive, Proceedings of the National Academy of Sciences, vol. 101, no. 13, pp. 4655-4660, 2004.

[5] J. Mejias and A. Longtin, Optimal heterogeneity for coding in spiking neural networks, Physical Review Letters, vol. 108, no. 22, 228102, 2012.

[6] N. Hiratani, J.-N. Teramae, and T. Fukai, Associative memory model with long-tail-distributed hebbian synaptic connections, Frontiers in computational neuroscience, vol. 6, 102, 2013.

[7] S. Nobukawa and H. Nishimura, Chaotic resonance in coupled inferior olive neurons with the llinás approach neuron model, Neural computation, vol. 28, no. 11, pp. 2505-2532, 2016.

[8] S. Nobukawa, H. Nishimura, and T. Yamanishi, Chaotic resonance in typical routes to chaos in the Izhikevich neuron model, Scientific reports, vol. 7, no. $1,1331,2017$.

[9] N. K. Kasabov, Neucube: A spiking neural network architecture for mapping, learning and understanding of spatio-temporal brain data, Neural Networks, vol. 52, pp. 62-76, 2014.

[10] J. H. Lee, T. Delbruck, and M. Pfeiffer, Training deep spiking neural networks using backpropagation, Frontiers in neuroscience, vol. 10, 508, 2016.

[11] X. Lin, X. Wang, and Z. Hao, Supervised learning in multilayer spiking neural networks with inner products of spike trains, Neurocomputing, vol. 237, pp. 59-70, 2017.

[12] S. R. Kulkarni and B. Rajendran, Spiking neural networks for handwritten digit recognitionsupervised learning and network optimization, Neural Networks, vol. 103, pp. 118-127, 2018.

[13] S. R. Kheradpisheh, M. Ganjtabesh, S. J. Thorpe, and T. Masquelier, STDP-based spiking deep convolutional neural networks for object recognition, Neural Networks, vol. 99, pp. 56-67, 2018.

[14] Z. Lin, D. Ma, J. Meng, and L. Chen, Relative ordering learning in spiking neural network for pattern recognition, Neurocomputing, vol. 275, pp. 94-106, 2018.

[15] A. Tavanaei, T. Masquelier, and A. Maida, Representation learning using event-based STDP, Neural Networks, vol. 105, pp. 294-303, 2018.

[16] M. Mozafari, S. R. Kheradpisheh, T. Masquelier, A. Nowzari-Dalini, and M. Ganjtabesh, First-spikebased visual categorization using reward-modulated STDP, IEEE Transactions on Neural Networks and Learning Systems, vol. 99, pp. 1-13, 2018.
[17] A. Tavanaei, Z. Kirby, and A. S. Maida, Training spiking convnets by STDP and gradient descent, in Proceedings of 2018 International Joint Conference on Neural Networks (IJCNN). IEEE, 2018, pp. 1-8.

[18] Y. Wu, L. Deng, G. Li, J. Zhu, and L. Shi, Spatio-temporal backpropagation for training highperformance spiking neural networks, Frontiers in neuroscience, vol. 12, 331, 2018.

[19] M. Bernardo, C. Budd, A. R. Champneys, and P. Kowalczyk, Piecewise-smooth dynamical systems: theory and applications. Springer Science \& Business Media, 2008, vol. 163.

[20] N. Kasabov, Neucube evospike architecture for spatio-temporal modelling and pattern recognition of brain signals, in Proceedings of IAPR Workshop on Artificial Neural Networks in Pattern Recognition. Springer, 2012, pp. 225-243.

[21] N. Kasabov and E. Capecci, Spiking neural network methodology for modelling, classification and understanding of EEG spatio-temporal data measuring cognitive processes, Information Sciences, vol. 294, pp. 565-575, 2015.

[22] C. Ge, N. Kasabov, Z. Liu, and J. Yang, A spiking neural network model for obstacle avoidance in simulated prosthetic vision, Information Sciences, vol. 399, pp. 30-42, 2017.

[23] D. Verstraeten, B. Schrauwen, D. Stroobandt, and J. Van Campenhout, Isolated word recognition with the liquid state machine: a case study, Information Processing Letters, vol. 95, no. 6, pp. 521-528, 2005.

[24] A. Ghani, T. M. McGinnity, L. P. Maguire, and J. Harkin, Neuro-inspired speech recognition with recurrent spiking neurons, in Proceedings of International Conference on Artificial Neural Networks. Springer, 2008, pp. 513-522.

[25] Z. Yanduo and W. Kun, The application of liquid state machines in robot path planning, Journal of Computers, vol. 4, no. 11, pp. 1183-1186, 2009.

[26] Y. Zhang, P. Li, Y. Jin, and Y. Choe, A digital liquid state machine with biologically inspired learning and its application to speech recognition, IEEE transactions on neural networks and learning systems, vol. 26, no. 11, pp. 2635-2649, 2015.

[27] Y. Jin and P. Li, Calcium-modulated supervised spike-timing-dependent plasticity for readout training and sparsification of the liquid state machine, in Proceedings of 2017 International Joint Conference on Neural Networks (IJCNN). IEEE, 2017, pp. 2007-2014. 
[28] R. V. Florian, Reinforcement learning through modulation of spike-timing-dependent synaptic plasticity, Neural Computation, vol. 19, no. 6, pp. 1468-1502, 2007.

[29] N. Frémaux, H. Sprekeler, and W. Gerstner, Functional requirements for reward-modulated spiketiming-dependent plasticity, Journal of Neuroscience, vol. 30, no. 40, pp. 13 326-13 337, 2010.

[30] T.-S. Chou, L. D. Bucci, and J. L. Krichmar, Learning touch preferences with a tactile robot using dopamine modulated STDP in a model of insular cortex, Frontiers in neurorobotics, vol. 9, p. 6, 2015.

[31] A. H. Marblestone, G. Wayne, and K. P. Kording, Toward an integration of deep learning and neuroscience, Frontiers in computational neuroscience, vol. 10, 94, 2016.

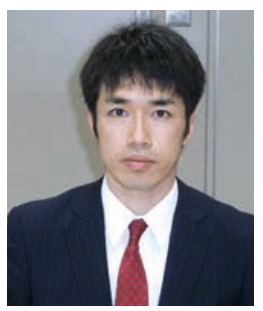

Sou Nobukawa graduated from the Department of Physics and Earth Sciences, University of Ryukyus in 2006, and completed the doctoral program at University of Hyogo in 2013. He is an Associate Professor at the Department of Computer Science, Chiba Institute of Technology. His research interests include chaos/bifurcation and neural networks. He is a member of IEEE, INNS, IEICE, IPSJ, SICE, ISCIE, and other organizations, and was awarded the SICE encouraging prize in 2016.

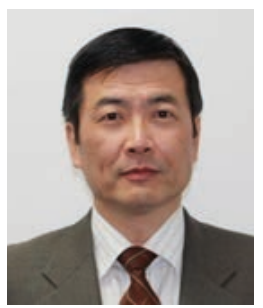

Haruhiko Nishimura graduated from the Department of Physics, Shizuoka University in 1980, and completed the doctoral program at Kobe University in 1985 . He is currently a Professor at the Graduate School of Applied Informatics, University of Hyogo. His research interests include intelligent
[32] A. S. Warlaumont and M. K. Finnegan, Learning to produce syllabic speech sounds via rewardmodulated neural plasticity, PloS one, vol. 11, no. 1, e0145096, 2016.

[33] Y. Kawai, T. Takimoto, J. Park, and M. Asada, Efficient reward-based learning through body representation in a spiking neural network, in Proceedings of the 8th Joint IEEE International Conference on Development and Learning and on Epigenetic Robotics. IEEE, 2018, pp. 198-203.

[34] E. M. Izhikevich, Polychronization: computation with spikes, Neural computation, vol. 18, no. 2, pp. 245-282, 2006.

[35] E. M. Izhikevich, Solving the distal reward problem through linkage of STDP and dopamine signaling, Cerebral cortex, vol. 17, no. 10, pp. 2443-2452, 2007.

systems science based on several architectures such as neural networks and complex systems. He is also currently engaged in research in biomedical, healthcare, and high-confidence sciences. He is a member of the IEEE, IEICE, IPSJ, ISCIE, JNNS, and other organizations, and was awarded the ISCIE paper prize in 2001 and JSKE paper prize in 2010.

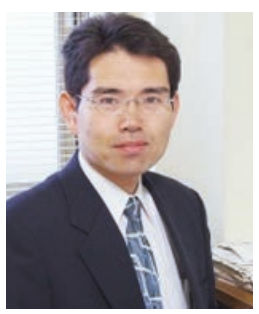

Teruya Yamanishi received a Master's degree in education of science from Kobe University in 1991, and a Ph.D. degree in physics from Kobe University in 1994. He is a Professor at Fukui University of Technology, where he studies mathematical information science for the brain, and develops optimization tools for behavior of autonomous robots. 